\title{
A psychological flexibility-based intervention for modulating the impact of stigma and prejudice: a descriptive review of empirical evidence
}

\author{
Akihiko Masuda*, Mary L. Hill, Jessica Morgan and Lindsey L. Cohen \\ Georgia State University
}

\begin{abstract}
In recent years, there have been growing efforts to understand and modulate stigma and prejudice from the standpoint of the psychological flexibility model, a pragmatic model of complex human behavior. The present paper provides an overview of the empirical evidence on the applicability of the psychological flexibility model, and its applied strategy, acceptance and commitment therapy (ACT), to stigma and prejudice. Preliminary findings suggest that the psychological flexibility model and ACT are promising avenues for reducing stigma and prejudice; however, further investigation and refinement of the model and ACT are crucial for significantly ameliorating human suffering related to stigma and prejudice.
\end{abstract}

Keywords: stigma, prejudice, acceptance and commitment therapy, psychological flexibility.

Una intervención psicológica basada en la flexibilidad para modular el impacto del estigma y prejuicio: una revisión descriptiva de la evidencia empirica.

RESUMEN: En los últimos años, se han producido crecientes esfuerzos por comprender y modular el estigma y los prejuicios desde la perspectiva del modelo de flexibilidad psicológica, un modelo pragmático de la conducta humana compleja. El presente artículo ofrece una visión general de la evidencia empírica sobre la aplicabilidad del modelo de flexibilidad psicológica, y su estrategia de aplicación, la terapia de aceptación y compromiso (ACT) en el estigma y los prejuicios. Los resultados preliminares sugieren que el modelo de flexibilidad psicológica y ACT son estrategias prometedoras para la reducción del estigma y el prejuicio; sin embargo, la investigación y el perfeccionamiento del modelo y de ACT son cruciales para mejorar significativamente el sufrimiento humano relacionado con el estigma y los prejuicios.

Palabras clave: estigma, prejuicio, Terapia de Aceptación y Compromiso, flexibilidad psicológica.

Stigma towards individuals based on their group membership is a major source of human despair. Prejudice and discrimination based on group categorization status is found across nearly all domains of society including employment, housing, education, health care, judicial systems, and financial systems (e.g., Pager $\&$ Shepherd, 2008). The verbal and sociocultural processes in these realms result in negative emotional and health consequences for the victims of discrimination 
based on race and ethnicity (Pascoe \& Richman, 2009), mental illness (Corrigan \& Penn, 1999; Link, 1987; Livingston \& Boyd, 2010), addiction (Luoma \& Kohlenberg, in press), HIV positive status (Gonzalez, Solomon, Zvolensky, $\&$ Miller, 2009), obesity (Puhl \& Heuer, 2009), and sexual minority status (Yadavaia \& Hayes, 2012). Given their significant impact, stigma and prejudice are important targets for reducing human suffering and improving the quality of life of people who have been socioculturally marginalized.

\section{PSYCHOLOGICAL FLEXIBILITY MODEL}

The psychological flexibility model (Hayes, Luoma, Bond, Masuda, \& Lillis, 2006 ) is a pragmatic theory of complex human behavior. The model is pragmatic in the sense that it aims not only to understand the behavioral phenomena of interest (e.g., stigma, prejudice), but also to influence them in order to move toward a specified goal (e.g., the reduction of stigmatization in society). This applied model is derived from relational frame theory (RFT; Hayes, Barnes-Holmes, \& Roche, 2001), and its principle-based intervention strategy is called acceptance and commitment therapy (ACT; Hayes, Strosahl, \& Wilson, 2012).

In recent years, the psychological flexibility model, originally developed as a conceptual framework for psychopathology and psychological health, has been applied to the issues related to stigma and prejudice (e.g., Hayes, Niccolls, Masuda, \& Rye, 2002; Lillis \& Hayes, 2007; Masuda et al., 2007; Masuda et al., 2009). Although research on the application of the psychological flexibility model and ACT to stigma and prejudice is still in its early stages, several studies have investigated its applicability to various forms of stigma and prejudice. As such, the purpose of the present paper is to review the literature that has specifically applied the psychological flexibility model and ACT to stigma and prejudice.

\section{PSYCHOLOGICAL FLEXIBILITY ACCOUNT OF STIGMA AND PREJUDICE}

The psychological flexibility model defines stigma and prejudice as generalized verbal processes that involve normal and adaptive human language/verbal abilities that have been "inappropriately" applied (Hayes et al., 2001; Lillis \& Levin, in press). More specifically, stigma and prejudice are roughly defined as the process of objectification and dehumanization of self or others as a result of their participation in normal verbal processes of categorization, association, and evaluation (Hayes et al., 2002). This broad definition includes bias and discrimination applied to any verbally categorized and evaluated groups of individuals (i.e., social categorization), such as "White," "gay," "Muslim," "woman," "poor," "addict," "handicapped," and so on. This definition also emphasizes how language/verbal processes make acts of bias and discrimination possible.

The normal verbal processes of categorizing, associating, and evaluating can occur in virtually every sociocultural context automatically and without cons- 
cious awareness (Hayes et al., 2002; Lillis \& Levin, in press). Unfortunately, this automaticity also applies to stigma and prejudice. For example, even individuals who deny prejudiced attitudes often maintain implicit (i.e., automatic, unconscious) racial biases (Greenwald, Poehlman, Uhlmann, \& Banaji, 2009). These automatic biases are important because, even if individuals are unconscious of them, they have the potential to subtly influence discriminatory behaviors in many ways (Dasgupta, 2004).

Stigma and prejudice are also inherently rigid (Haghighat, 2001; Hayes et al., 2002; Kurzban \& Leary, 2001). New ideas are met with resistance when they are not consistent with stereotype-consistent beliefs (Moxon, Keenan, \& Hine, 1993), and efforts to suppress unwanted thoughts often paradoxically increase their frequency and intensity (Wenzlaff \& Wegner, 2000). As previously mentioned, implicit thoughts occur automatically and potentially without awareness (Greenwald \& Banaji, 1995). Stigmatization and prejudice may also be adaptive and pervasive in part because they allow one to more easily navigate complex sociocultural interactions (Macrae, Milne, \& Bodenhausen, 1994). This arbitrary categorization and association is learned early in childhood and continues throughout one's lifetime (Hayes et al., 2001). Given the pervasive and rigid nature of stigma and prejudice, some researchers have questioned the feasibility of directly changing stigmatizing and prejudicial thoughts in form and frequency (Bargh, 1999; Wilson, Lindsey, \& Schooler, 2000).

It should be noted that various forms of stigma and prejudice, though differing in content, may not be qualitatively distinct from one another in process (Lillis \& Levin, in press). According to the psychological flexibility model, stigma and prejudice are defined as a general process of having biases and engaging in discrimination towards individuals based on arbitrarily evaluative categories, regardless of their particular topographic form (e.g., racism vs. sexism; Hayes et al., 2002). This conceptual position is supported in part by the finding that prejudiced attitudes towards various groups tend to co-occur and comprise a single latent variable (e.g. Bäckström \& Björklund, 2007; Lillis \& Levin, in press). For example, individuals who are prejudiced toward African Americans are also likely to be biased against other groups, such as ethnic minorities, women, and sexual minorities (Akrami, Ekehammar, \& Bergh, 2011). As such, targeting the verbal processes that underlie stigma and prejudice, instead of focusing on the content of beliefs and biases towards specific groups, may be an effective method to undermine the negative impact of stigma and prejudice (Lillis \& Levin, in press).

\section{PSYCHOLOGICAL FLEXIBILITY-BASED INTERVENTIONS FOR MODU- LATING THE IMPACT OF STIGMA AND PREJUDICE}

The salient features of an ACT intervention for stigma and prejudice are its focus on (a) the underlying verbal processes of categorization, association, and evaluation rather than the specific topographical content of stigmatizing 
thoughts; and (b) the promotion of intrinsic and prosocial actions alternative to or incompatible with stigmatization and discrimination, rather than directly challenging and making efforts to refute stigma and prejudice (Hayes et al., 2004; Masuda et al., 2009). Interestingly, emerging trends within the literature on stigma and prejudice interventions have begun to highlight the conceptual and practical relevance of the psychological flexibility processes that are targeted by ACT interventions (also see Lillis \& Levin, in press).

Discouraging social pressure and thought suppression. One method of reducing stigma and prejudice is via social influence (for a review, see Corrigan \& Penn, 1999). For instance, expert opinions (e.g., Levy, Stroessner \& Dweck, 1998), protest (Corrigan \& Penn, 1999), and social norms messages (e.g., Stangor, Sechrist, \& Jost, 2001) can all counter prejudice. Unfortunately, the literature suggests that when external motivators (i.e., social pressure) are used, it can result in increases in stigma and prejudice (e.g., Legault, Gutsell, \& Inzlicht, 2011).

External pressure might lead to heightened stigma and prejudice in part because it promotes ineffective suppression strategies (Hausmann \& Ryan, 2004). There are ample data to suggest that thought suppression can have paradoxical effects (e.g., Wenzlaff \& Wegner, 2000). Thus, when people are pressured to not stigmatize and refrain from prejudiced thoughts, they may attempt suppression and cognitive control strategies. These efforts will likely lead to subsequent increases in these thoughts and possibly related behavior (e.g., Galinsky \& Moskowitz, 2000). Thus, it is important to explore other methods of reducing stigma and prejudice that do not encourage thought suppression. For example, an ACT approach might highlight values-directed behaviors and acceptance of and defusion from prejudiced thoughts (Hayes et al., 2004).

Enhancing internal motivation. Researchers have evaluated whether increasing personally relevant motivation effectively reduces stigma and prejudice. Data suggest that increases in internal motivation are related to decreases in stigma and prejudice (Legault, Green-Demers \& Eadie, 2009). Further, interventions targeting internal motivation have resulted in lower explicit as well as implicit prejudice (Legault et al., 2011). In short, the data suggest that, enhancing motivation linked to self-selected, personally-relevant prosocial goals and values may be an effective method to reduce stigma and prejudice (Masuda et al., 2009).

Increasing awareness of automatic stigma and prejudices. Subtle forms of prejudice are distinguished in that individuals deny explicit prejudiced beliefs, but demonstrate implicit biases (Todd, Bodenhausen, Richeson, \& Galinsky, 2011). Possibly, a lack of awareness or an unwillingness to acknowledge one's prejudices leads to a discrepancy between explicit and implicit beliefs. Thus, raising awareness about this disconnect might be a first step in treatment (Monteith $\&$ Mark, 2005). In fact, a study showed that encouraging awareness of prejudice 
reduced discriminatory behavior among individuals with low explicit and high implicit prejudice (Son Hing, Li, \& Zanna, 2002). Similarly, increased awareness of one's own biases is a cornerstone of multicultural competency training (Sue, Zane, Hall, \& Berger, 2009).

Promoting cognitive flexibility, perspective-taking, and empathy. There is a rich literature documenting prejudice reduction success via altering the favoring of perceived in-groups over out-groups by targeting the salience of particular group statuses (Masuda et al., 2009; Paluck \& Grenn, 2009). These interventions elaborate perceived group statuses and directly target the emphasis on "us" vs. "them." For example, perspective-taking manipulations such as writing about or imagining what someone else - someone who belongs to a marginalized group - might be thinking and feeling can increase empathy and reduce explicit and implicit prejudice (e.g., Galinsky \& Moskowitz, 2000; Shih, Wang, Bucher, \& Stotzer, 2009). These in- and out-group prejudices may be reduced through encouraging identification with an over-arching category that places both individuals in the same group. One example of this approach is compassion-focused interventions designed to foster the sense of commonality in suffering (Fredrickson, Cohn, Coffey, Pek, \& Finkel, 2008). It appears that the mechanism of change for compassion-focused interventions is via increasing self-other overlap and highlighting similarities in important domains (Galinsky et al., 2005).

Increasing contact. Avoidance is a key aspect of stigma and prejudice; marginalized groups and individuals are often socially isolated (Markowitz, 1998). Thus, a common approach to stigma and prejudice reduction is simply to increase contact between the individuals with prejudice and those in marginalized groups (Corrigan \& Penn, 1999). A meta-analysis of 515 studies showed that increased contact resulted in reducing prejudice (Pettigrew \& Tropp, 2006). Results were particularly strong when contact occurred under certain conditions including equality, cooperation, authority support, and a shared goal (Pettigrew \& Tropp, 2006). Research suggests that increased liking, empathy, and self-disclosure as well as decreased intergroup anxiety may explain how intergroup contact reduces prejudice (e.g., Turner, Hewstone, \& Voci, 2007). Further, personal importance of contact is a key variable in predicting whether increased contact leads to prejudice reduction (Van Dick et al., 2004).

Summary. This review touches on approaches to stigma and prejudice reduction, which are consistent with a psychological flexibility model. It is critical that researchers and clinicians continue to explore methods of reducing prejudiced thoughts, motivation, and behavior Emerging data suggest that key approaches are teaching awareness and flexibility with prejudiced thoughts, discouraging thought suppression strategies, emphasizing internal motivation for prejudice reduction rather than external motivation, promoting perspective-taking, and encouraging intergroup interactions situated within a cooperative, prosocial con- 
text. We now turn to a discussion of the empirical evidence of ACT interventions for stigma and prejudice.

\section{ACCEPTANCE AND COMMITMENT THERAPY FOR STIGMA AND PREJUDICE}

As stated earlier, several preliminary studies have investigated the applicability of psychological flexibility-based interventions (i.e., ACT) to a number of types of stigma and prejudice. These include treatments targeting stigma against others - public stigma toward mental illness, racial prejudice, and addiction counselor stigma toward clients - and interventions targeting stigma against the self - weight-related self-stigma, self-stigma in addiction, and self-stigma related to same-sex attraction. Conceptually, a flexibility-based intervention can be implemented using various formats (e.g., individual, group, and workshop). However, the majority of ACT interventions for reducing stigma and prejudice have been administered in a workshop format (e.g., one-day group). In the literature, the workshop format of ACT is sometimes termed acceptance and commitment training (ACT; Hayes et al., 2004) - as opposed to acceptance and commitment therapy - in order to differentiate it from an individual and group psychotherapy format of ACT. As stigma and prejudice are often categorized based on their direction (e.g., stigma toward others vs. self), this section presents ACT empirical studies separately for public stigma and prejudice (stigmatizing attitudes toward others) and self-stigma (internalized stigma).

\section{PUBLIC STIGMA AND PREJUDICE}

The first ACT study on stigma (Hayes et al., 2004) investigated the effects of ACT on licensed addictions counselors' stigmatizing attitudes toward their clients. In the study, 90 licensed addictions counselors were randomly assigned to attend a one-day (6-hour) workshop based on ACT training $(n=30)$, Multicultural Training $(n=30)$, or a control lecture focused on a biological basis for methamphetamine addiction $(n=30)$. The ACT-based workshop consisted of both didactic and experiential exercises, which were drawn from the original ACT manual (Hayes et al., 1999).

The ACT workshop emphasized the view that stigmatization is built on human language processes. In the workshop, participants were encouraged to notice the automatic nature of stigmatizing processes (i.e., verbal categorization, association, and evaluation) and learned acceptance and mindfulness skills (see Hayes et al., 2012) to reduce the impact and believability of stigmatizing attitudes and negative self-referent thoughts (e.g., shame) even if they continue to occur. Several exercises were used deliberately to elicit difficult emotions and thoughts about clients and self, and the group practiced experiencing these internal responses without making efforts to alter them in form or frequency. Finally, participants were guided through the nature and importance of values and were 
encouraged to choose value-consistent actions (e.g., to act on the intrinsic value of helping others).

Results revealed that stigmatizing attitudes were reduced post-training in both active treatment groups, but only the ACT condition had lower scores at the three-month follow- up. The ACT intervention also decreased professional burnout at the three-month follow up, suggesting that interventions targeting stigma by providers may also have the effect of promoting their well-being and professional effectiveness.

The second ACT study investigated the effects of a briefer ACT workshop targeting mental health stigma (i.e., stigmatizing attitudes toward people with a mental disorder) in a non-clinical undergraduate sample (Masuda et al., 2007). In the study, 95 undergraduates were randomly assigned to either a 2.5-hour onetime ACT workshop or an education-based workshop of the same length. The ACT protocol was largely drawn from the original ACT manual (Hayes et al., 1999) as well as the ACT protocol used in Hayes et al. (2004), with only minor modifications of intervention techniques in order to specifically target mental health stigma. Once again, workshop leaders emphasized the view that stigma is built into our normal use of language, and that the solution to these processes is more likely to be found in compassion toward self and others and values-guided behaviors than in the reduction of the form and frequency of stigmatizing attitudes.

Results revealed that the effects of these interventions were moderated by participants' psychological flexibility at pre-intervention. That is, for those high in psychological flexibility at pre-intervention, both interventions were equally successful in reducing mental health stigma at post-intervention and one-month follow-up. However, only the ACT group significantly reduced mental health stigma in those with lower levels of psychological flexibility. These findings suggest that ACT interventions are broadly applicable regardless of participants' levels of psychological flexibility, but are particularly helpful for those who are less psychologically flexible. A subsequent analysis using the results of an open trial (Masuda et al., 2009) of the same intervention with 22 undergraduates also showed that change scores of psychological flexibility from pre-intervention to one-month follow-up were correlated with change scores of stigmatizing attitudes within the same time period. That is, the magnitude of change in psychological flexibility was significantly related to the magnitude of change in stigmatizing beliefs one month after the intervention.

Employing a counterbalanced within-group design, Lillis and Hayes (2007) investigated the effect of ACT, relative to an education condition, on racial prejudice. A total of 32 undergraduates (13 men, 19 women; 11 U.S. ethnic minority), who were enrolled in two separate classes on racial differences, were exposed to each approach in a counterbalanced order. Each approach was 75-minutes in duration. Once again, the ACT workshop was designed based on the ACT stigma protocol (Hayes et al., 2004) and was specifically tailored to the topic of racial and ethnic prejudice. Discussions and experiential exercises in the workshop 
were designed to assist the participants (a) to become mindfully aware of their own prejudicial thoughts and feelings and reactions, (b) to accept those thoughts and feelings as the natural result of learning and using language in a prejudicial society, (c) to notice the automatic processes of evaluation and judgment more generally, and (d) to orient to positive actions consistent with their own values regarding how to treat other human beings. Only the ACT intervention was effective in increasing positive behavioral intentions at post and a 1-week follow-up. These changes were associated with other self-reported changes that fit with the ACT model, such as increased awareness and acknowledgement of bias, acceptance, and flexibility.

\section{SELF-STIGMA AND PREJUDICE}

ACT interventions have also been effective in targeting stigma directed toward the self. Lillis, Hayes, Bunting, and Masuda (2009) randomly assigned 84 patients who had completed a weight loss program to either a waitlist or a one-day, 6-hour psychological flexibility-based workshop (ACT) targeting obesity-related stigma and psychological distress. The ACT intervention included exercises and materials from the original ACT book (Hayes et al., 1999) as well as an ACT stigma protocol (Hayes et al., 2004). Each workshop, led by two ACTtrained facilitators, employed a structured sequence of lectures and exercises. Specific methods taught included acceptance, mindfulness, and defusion skills applied to difficult thoughts, feelings, and bodily sensations more generally, with a particular focus on primarily on weight-related stigmatizing thoughts and distress. The workshop also focused heavily on the participants' life values, especially those related to health and relationships. More specifically, the facilitators and the workshop members identified barriers to their values-consistent behaviors (e.g., self-stigma, shame) and fostered motivation and behavioral commitments to values-consistent living. A general ACT self-help workbook (Hayes \& Smith, 2005) was also distributed to participants to encourage further implementation of the techniques they learned. Neither the workshop nor the workbook contained strategies for losing weight, and no weight loss goals or strategies were set during the workshop. The goal of the workshop was presented to participants as "living a more fulfilling life consistent with your chosen values." Nevertheless, at the three-month follow-up, the ACT group showed greater improvements in body mass, quality of life, psychological distress, and weight-related stigma, than the control group. Changes in weight-specific acceptance and psychological flexibility mediated the changes in these outcomes.

ACT has also been examined in targeting self-stigma among patients with substance use difficulties. An open trial first examined ACT in a sample of 48 adults with substance use disorders in a residential setting (Luoma, Kohlenberg, Hayes, Bunting, \& Rye, 2008). The ACT protocol on average consisted of three 2-hour group workshops within the same week, focusing on using the processes of psychological acceptance, cognitive defusion, and contact with important 
values to help participants learn to respond to their stigmatizing thoughts and behaviors in a way that would not obstruct recovery. This trial resulted in improvements across a range of measures at post-treatment, including reduced internalized shame, higher self-esteem, and greater psychological flexibility.

Subsequently, a larger pilot study (Luoma, Kohlenberg, Hayes, \& Fletcher, 2012) investigated the effects of an ACT group workshop on self-stigma and shame among patients with addictions who were attending a 28-day addiction residential treatment program. In this study, 133 addiction treatment patients were randomly assigned to either a 6-hour ACT group protocol (three 2-hour sessions within a single week; Luoma et al., 2008) or to treatment as usual (TAU). The ACT intervention replaced six hours of the usual treatment of the 28-day residential program. At post-treatment, outcomes for ACT were not promising. Those in the ACT group showed smaller reductions in shame at post-treatment than the TAU group, and equivalent increases in general mental health, quality of life, and total social support. However, ACT outperformed TAU at 4-month followup. Participants in the ACT condition showed numerous improvements over the follow-up period, including reduced shame, increased general mental health, increased quality of life, and increased social support. In addition, they reported more treatment attendance and fewer days of substance use over the follow-up period. The TAU group, however, deteriorated over the follow-up period on measures of internalized shame, general mental health, and quality of life. This study also suggests that targeting self-stigma and shame is beneficial in influencing global improvements in substance abusing populations. In addition, effects of the ACT intervention on treatment utilization at follow-up were statistically mediated by post-treatment levels of shame, in that those evidencing higher levels of shame at post-treatment were more likely to be attending treatment at follow-up if they received ACT. This finding is important as intervention effects on substance use at follow-up were also found to be mediated by treatment utilization at follow-up.

Finally, a multiple-baseline study by Yadavaia and Hayes (2012) evaluated a 6-10 session ACT intervention for self-stigma related to same-sex attraction in five adults who self-identified as "being a sexual minority." All of the five participants showed sizeable reductions in the degree to which thoughts about sexual attraction interfered with their lives and the distress associated with those thoughts at post-treatment and at 4- and 12-week follow-up. Positive changes were also observed in several psychosocial outcomes, including internalized homophobia, depression, anxiety, stress, quality of life, and perceived social support. Together, these studies provide preliminary evidence for interventions based on a psychological flexibility model in reducing self-stigma.

\section{CONCLUSION}

Despite the large burden of stigma and prejudice on society, few empirically supported interventions have been developed to help those living under their 
weights. More traditional methods focusing on first-order cognitive change (i.e., direct attempts to alter stigmatizing thoughts) have generally shown limited effectiveness and have not led to robust programs of implementation. Recent developments based on the psychological flexibility model focus on second-order cognitive strategies and have closer connections to basic research on the nature of stigma and stereotyping. Whereas early ACT intervention trials suggest that this approach is promising, it is important to further refine and test its conceptual applicability so that treatment might be eventually disseminated broadly.

\section{REFERENCES}

Akrami, N., Ekehammar, B., \& Bergh, R. (2011). Generalized prejudice: Common and specific components. Psychological Science, 22(1), 57-59. doi:10.1177/0956797610390384

Bäckström, M. \& Björklund, F. (2007). Structural modeling of generalized prejudice: The role of social dominance, authoritarianism, and empathy. Journal of Individual Differences, 28, 10-17.

Bargh, J. A. (1999). The cognitive monster: The case against the controllability of automatic stereotype effects. In S.Chaiken \& Y.Trope (Eds.), Dual process theories in social psychology (pp. 361-382). New York, NY

Corrigan, P. W., \& Penn, D. L. (1999). Lessons from social psychology on discrediting psychiatric stigma. American Psychologist, 54(9), 765-776

Dasgupta, N. (2004). Implicit ingroup favoritism, outgroup favoritism, and their behavioral manifestations. Social Justice Research, 17, 143-169.

Fredrickson, B. L., Cohn, M. A., Coffey, K. A., Pek, J., \& Finkel, S. M. (2008). Open hearts build lives: Positive emotions, induced through loving-kindness meditation, build consequential personal resources. Journal of Personality and Social Psychology, 95(5), 1045-1062.

Galinsky, A. D., Ku, G., \& Wang, C. S. (2005). Perspective-taking and self-other overlap: Fostering social bonds and facilitating social coordination. Group Processes \& Intergroup Relations, 8(2), 109-124.

Galinsky, A. D., \& Moskowitz, G. B. (2000). Perspective-taking: Decreasing stereotype expression, stereotype accessibility, and ingroup favoritism. Journal of Personality and Social Psychology, 78, 708-724.

Greenwald, A. G., \& Banaji, M. R. (1995). Implicit social cognition: Attitudes, self-esteem, and stereotypes. Psychological Review, 102, 4-27.

Gonzalez, A., Solomon, S. E., Zvolensky, M. J., \& Miller, C. T. (2009). The interaction of mindful-based attention and awareness and disengagement coping with HIV/AIDS-related stigma in regard to concurrent anxiety and depressive symptoms among adults with HIV/AIDS. Journal of Health Psychology, 14, 403-413.

Greenwald, A. G., Poehlman, T. A., Uhlmann, E. L., \& Banaji, M. R. (2009). Understanding and using the Implicit Association Test: III. Meta-analysis of predictive validity. Journal of Personality and Social Psychology, 97, 17-41. 
Haghighat, R. (2001). A unitary theory of stigmatization: Pursuit of self-interest and routes to destigmatisation. British Journal of Psychiatry, 178, 207-215.

Hausmann, L.R.M. \& Ryan, C.S. (2004). Effects of external and internal motivation to control prejudice on implicit prejudice: The mediating role of efforts to control prejudiced responses. Basic and Applied Social Psychology, 26, 215-225.

Hayes, S. C., Barnes-Holmes, D., \& Roche, B. (2001). Relational frame theory: A post-Skinnerian account of human language and cognition. New York, NY US: Kluwer Academic/Plenum Publishers.

Hayes, S. C., Bissett, R., Roget, N., Padilla, M., Kohlenberg, B. S., Fisher, G., et al. (2004). The impact of acceptance and commitment training and multicultural training on the stigmatizing attitudes and professional burnout of substance abuse counselors. Behavior Therapy, 35(4), 821-835.

Hayes, S. C., Luoma, J. B., Bond, F. W., Masuda, A., \& Lillis, J. (2006). Acceptance and commitment therapy: Model, processes and outcomes. Behaviour Research And Therapy, 44(1), 1-25. doi

Hayes, S. C., Niccolls, R., Masuda, A., \& Rye, A. K. (2002). Prejudice, terrorism and behavior therapy. Cognitive and Behavioral Practice, 9(4), 296-301.

Hayes, S. C., Strosahl, K. D., \& Wilson, K. G. (1999). Acceptance and commitment therapy: An experiential approach to behavior change. New York, NY US: Guilford Press.

Hayes, S. C., Strosahl, K. D., \& Wilson, K. G. (2012). Acceptance and commitment therapy: The process and practice of mindful change (2nd ed.). New York, NY US: Guilford Press.

Kurzban, R., \& Leary, M. R. (2001). Evolutionary origins of stigmatization: The functions of social exclusion. Psychological Bulletin, 127,187-208.

Legault, L., Green-Demers, I., \& Eadie, A. L. (2009). When internalization leads to automatization: The role of self-determination in automatic stereotype suppression and implicit prejudice regulation. Motivation and Emotion, 33, $10-24$.

Legault, L., Gutsell, J. N., \& Inzlicht, M. (2011). Ironic effects of antiprejudice messages: How motivational interventions can reduce (but also increase) prejudice. Psychological Science, 22, 1472-.1477.

Levy, S. R., Stroessner, S. \& Dweck, C. S. (1998). Stereotype formation and endorsement: the role of implicit theories. Journal of Personality and Social Psychology, 74, 421-36

Lillis, J., \& Hayes, S. C. (2007). Applying acceptance, mindfulness, and values to the reduction of prejudice - A pilot study. Behavior Modification, 31, 389411.

Lillis, J., Hayes, S. C., Bunting, K., \& Masuda, A. (2009). Teaching Acceptance and Mindfulness to Improve the Lives of the Obese: A Preliminary Test of a Theoretical Model. Annals of Behavioral Medicine, 37(1), 58-69.

Lillis, J., \& Levin, M. (in press). Acceptance and mindfulness for undermining prejudice. In A. Masuda (Ed.), Mindfulness and acceptance in multicultural 
competency: A contextual approach to sociocultural diversity in theory and practice. Oakland, CA: New Harbinger.

Link, B. G. (1987). Understanding labeling effects in the area of mental disorders: An assessment of the effects of expectations of rejection. American Sociological Review, 52, 96-112.

Livingston, J. D., \& Boyd, J. E. (2010). Correlates and consequences of internalized stigma for people living with mental illness: a systematic review and meta-analysis. Social science \& medicine (1982), 71(12), 2150-61.

Luoma, J. B. (in press). Acceptance and mindfulness for undermining stigma. In A. Masuda (Ed.), Mindfulness and acceptance in multicultural competency: A contextual approach to sociocultural diversity in theory and practice. Oakland, CA: New Harbinger.

Luoma, J. B., \& Kohlenberg, B. S. (in press). Self-Stigma and Shame in Addictions. In Steven C. Hayes \& M. E. Levin (Eds.), Acceptance, Mindfulness, Values, and Addictive Behaviors: Counseling with Contemporary Cognitive Behavioral Therapies. Oakland, CA: New Harbinger.

Luoma, J. B., Kohlenberg, B. S., Hayes, S. C., Bunting, K., \& Rye, A. K. (2008). Reducing self-stigma in substance abuse through acceptance and commitment therapy: Model, manual development, and pilot outcomes. Addiction Research \& Theory, 16(2), 149-165.

Luoma, J.B., Kohlenber, B.S., Hayes, S.C., \& Fletcher, L. (2012). Slow and steady wins the race: A randomized clinical trial of acceptance and commitment therapy targeting shame in substance use disorders. Journal of Consulting and Clinical Psychology 80(1), 43-53.

Macrae, C. N., Milne, A. B., \& Bodenhausen, G. V. (1994). Stereotypes as energy-saving devices: A peek inside the cognitive toolbox. Journal of Personality and Social Psychology, 66, 37-47.

Markowitz, F. (1998). The effects of stigma on the psychological well-being and life satisfaction of persons with mental illness. Journal of Health and Social Behavior, 39, 335-347.

Masuda, A., Hayes, S. C., Fletcher, L. B., Seignourel, P. J., Bunting, K., Herbst, S. A., Twohig, M. P., et al. (2007). Impact of acceptance and commitment therapy versus education on stigma toward people with psychological disorders. Behaviour Research and Therapy, 45(11), 2764-2772.

Masuda, A., Hayes, S. C., Lillis, J., Bunting, K., Herbst, S. A., \& Fletcher, L. B. (2009). The relation between psychological flexibility and mental health stigma in Acceptance and Commitment Therapy: A preliminary process investigation. Behavior and Social Issues, 18(1), 1-16.

Monteith, M. J., \& Mark, A. Y. (2005). Changing one's prejudice ways: Awareness, affect, and self-regulation. European Review of Social Psychology, 16, 113-154.

Moxon, P. D., Keenan, M., \& Hine, L. (1993). Gender-role stereotyping and stimulus equivalence. Psychological Record, 43, 381-394.

Pager, D. \& Shepherd, H. (2008). The sociology of discrimination: Racial dis- 
crimination in employment, housing, credit, and consumer markets. Annual Review of Sociology, 34, 181-209.

Paluck, E. L., \& Green, D. P. (2009). Prejudice reduction: What works? A critical look at evidence from the field and the laboratory. Annual Review of Psychology, 60, 339-367.

Pascoe, E. A. \& Richman, L. S. (2009). Perceived discrimination and health: A meta-analytic review. Psychological Bulletin, 135, 531-554.

Pettigrew, T. F. \& Tropp, L. R. (2006). A meta-analytic test of intergroup contact theory. Journal of Personality and Social Psychology, 90, 751-783.

Puhl, R. M., \& Heuer, C. A. (2009). The stigma of obesity: A review and update. Obesity, 17(5), 941-964.

Shih, M., Wang, E., Bucher, A. T. \& Stotzer, R. (2009). Perspective taking: Reducing prejudice towards general outgroups and specific individuals. Group Processes \& Intergroup Relations, 12, 565-577.

Son Hing, L. S., Li, W. \& Zanna, M. P. (2002). Inducing hypocrisy to reduce prejudicial responses among aversive racists. Journal of Experimental Social Psychology, 381, 71-78.

Stangor, C., Sechrist, G. B. \& Jost, J. T. (2001). Changing racial beliefs by providing consensus information. Personality and Social Psychology Bulletin, 27, 484-94.

Sue, S., Zane, N., Hall, G. C. N., \& Berger, L. K. (2009). The case for cultural competency in psychotherapeutic interventions. Annual Review of Psycholo$g y, 60,525-548$.

Todd, A. R., Bodenhausen, G. V., Richeson, J. A., \& Galinsky, A. D. (2011). Perspective taking combats automatic expressions of racial bias. Journal of Personality and Social Psychology, 100, 1027-1042.

Turner, R. N., Hewstone, M. \& Voci, A. (2007). Reducing explicit and implicit outgroup prejudice via direct and extended contact: The mediating role of self-disclosure and intergroup anxiety. Journal of Personality and Social Psychology, 93, 369-388.

Van Dick, R., Wagner, U., Pettigrew, T. F., Christ, O., Wolf, C., Petzel, T., et al. (2004). The role of perceived importance in intergroup contact. Journal of Personality and Social Psychology, 87, 211-227.

Wenzlaff, R. M. \& Wegner, D. M. (2000). Thought suppression. Annual Review of Psychology, 51, 59-91.

Yadavaia, J. E., \& Hayes, S. C. (2012). Acceptance and commitment therapy for self-stigma around sexual orientation: A multiple baseline evaluation. Cognitive and Behavioral Practice, 19, 545-559.

Wilson, T. D., Lindsey, S., \& Schooler, T. Y. (2000). A model of dual attitudes. Psychological Review, 107, 101-126. 